\title{
Tratamento cirúrgico do traumatismo raquimedular por arma branca retida em coluna cervical: relato de caso
}

\section{Surgical Treatment for Spinal Cord Injury Caused by Knife Held in Cervical Spine: Case Report}

\footnotetext{
${ }^{1}$ Neurocirurgião do Hospital de Urgências de Goiânia (HUGO), Goiânia, GO, Brasil.

2 Residente de Neurocirurgia do Hospital Geral de Goiânia (HGG), Goiânia, GO, Brasil.

${ }^{3}$ Acadêmico de Medicina da Pontifícia Universidade Católica de Goiás (PUC-GO), Goiânia, GO, Brasil.

${ }^{4}$ Neurocirurgião do Serviço de Neurocirurgia e do Departamento de Cirurgia da Coluna Vertebral do HGG, Goiânia, GO, Brasil.
}

Bernardo Drummond Braga ${ }^{1}$ Thiago Dantas de Souza Azarias ${ }^{2}$ Ariana Costa Cadurin ${ }^{3}$

Diego José Fernandes ${ }^{3}$ Oliver Vilela Gomes ${ }^{3} \quad$ Carlos Roberto S.A. Drummond ${ }^{4}$

\begin{abstract}
Address for correspondence Bernardo Drummond Braga, MD, rua T-29, 358, sala 603-setor Bueno, Goiânia, GO, Brasil CEP: 74210-050 (e-mail: bernardodrummond@yahoo.com.br).
\end{abstract}

Arq Bras Neurocir 2015;34:245-249.

\section{Resumo \\ Palavras-Chave \\ - traumatismos da medula espinhal \\ - ferimentos perfurantes \\ - síndrome de Brown- Séquard \\ - coluna cervical \\ - laminectomia \\ - prognóstico}

As lesões traumáticas da medula espinhal provocadas por objetos perfurantes retidos são raras e configuram um desafio para o cirurgião da coluna vertebral. A cirurgia precoce pode minimizar o risco de sequela neurológica. Exames de diagnósticos por imagem são fundamentais para o planejamento e sucesso terapêutico, mas não devem retardar o tratamento cirúrgico. Os autores descrevem um caso de lesão medular por arma branca retida provocando a síndrome de Brown-Séquard e discutem a abordagem terapêutica. Os objetivos da cirurgia são a retirada do objeto retido, sem causar déficit motor ou sensitivo adicional, e a correção da lesão dural e de eventuais instabilidades da coluna.

\footnotetext{
Abstract

Keywords

- spinal cord injuries

- stab

- Brown-Séquard syndrome

- laminectomy

- prognosis
}

Traumatic spinal cord injuries caused by perforating retained objects are rare and challenging for spinal surgeons. Early surgery can minimize the risk of neurological sequelae. Diagnostic imaging exams are essential for planning and therapeutic success, but should not delay surgical treatment. The authors describe a case of spinal cord injury by stab retained causing Brown-Sequard syndrome and discuss the therapeutic approach. The goal of surgery is the removal of the object retained without causing additional motor or sensory deficit, fix the dural injury and possible spinal instabilities.
Recebido

June 6, 2014

Aprovado

June 12, 2015
DOI http://dx.doi.org/ 10.1055/s-0035-1559889. ISSN 0103-5355.
Copyright $(\underset{0}{ } 2015$ by Thieme Publicações License terms Ltda, Rio de Janeiro, Brazil 


\section{Introdução}

O traumatismo raquimedular (TRM) está entre as principais causas de incapacidade física permanente no mundo. A prevalência é de 2,8 milhões de pacientes. Elevados custos econômicos e sociais estão implicados. ${ }^{1}$ Adultos jovens do sexo masculino são os mais afetados. Lesões neurológicas ocorrem em 15 a $20 \%$ dos casos. ${ }^{2}$

Ferimentos por armas brancas (FAB) são causas incomuns de TRM. Respondem por $1,5 \%$ do total. ${ }^{3}$ As lesões localizam-se predominantemente na região torácica (74\%), seguida pela coluna cervical (18\%) e lombar ( $8 \%)$. A penetração no sentido posterior-anterior ocorre na maioria dos casos. $^{8-11}$

Os déficits neurológicos que se instalam imediatamente após o TRM perfurante ocorrem por lesão direta pelo corpo estranho, por compressão de fragmento ósseo deslocado para o canal medular ou por isquemia secundária a lesão vascular.9,12,13

0 presente relato de caso objetiva apresentar o quadro clínico característico da lesão penetrante, as opções terapêuticas e os resultados do tratamento cirúrgico precoce de um paciente com TRM por arma branca retida na coluna cervical.

\section{Relato de Caso}

Paciente do sexo masculino de 34 anos foi admitido no Hospital de Urgências de Goiânia, vítima de agressão física com arma branca retida em coluna cervical (-Fig. 1).

Apresentava-se hemodinamicamente estável, com vias aéreas pérvias, respirando espontaneamente, sem dor abdominal, em decúbito ventral, imobilizado e com uma faca retida em coluna cervical. Ao exame neurológico, verificouse escala de coma de Glasgow de 15 , força de grau $\mathrm{V}$ em membro inferior direito e plegia em membro inferior esquerdo. Apresentava nível motor em C6 à esquerda, com força de grau III e sem déficit à direita. Sensibilidade termoálgica abolida à direita e preservada à esquerda. Sensibilidade vibratória e cinético-postural abolida à esquerda e preservada à direita. Ausência de nível sensitivo. 0 paciente

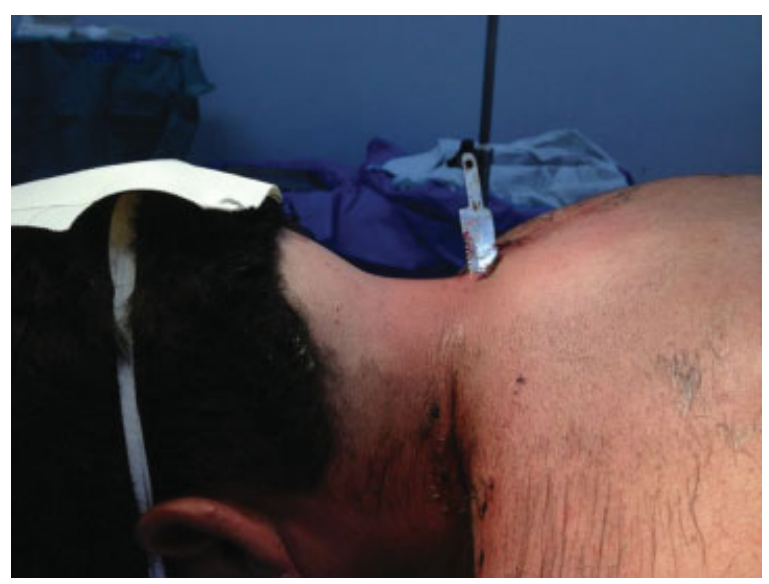

Fig. 1 Ferimento por arma branca retida em coluna cervical. não apresentava sinais clínicos de isquemia do território vertebrobasilar.

O exame de raios $\mathrm{X}$ da coluna cervical realizado antes da transferência do paciente mostrava a arma branca no nível de C6-C7 (-Fig. 2).

Foi solicitada tomografia da coluna cervical que mostrou a arma branca atravessando o canal vertebral no nível de C6-C7 até o forame da artéria vertebral à esquerda (-Fig. 3). A tomografia do crânio não apresentava alterações.

Após avaliação clínica e radiológica, o paciente foi encaminhado ao centro cirúrgico. Não foi realizado estudo dirigido para diagnóstico de lesão da artéria vertebral no préoperatório. $\mathrm{O}$ paciente foi submetido a anestesia geral, posicionado em decúbito ventral, e foi realizada antissepsia. Antibióticos profiláticos e dexametasona foram administrados antes do procedimento. Foi realizada incisão mediana e dissecção até a exposição das lâminas de C5 a C7 e visualização do corpo estranho perfurando a lâmina de C6.

Foi realizada pequena laminectomia de C7 até C6, preservando as facetas articulares, expondo a falha dural ao redor da arma branca. No final da laminectomia, a arma branca se encontrava solta, sem resistência para sua retirada (-Fig. 4). Realizada hemostasia e sutura da dura-máter com prolene e enxerto de fáscia muscular. Um dreno de sucção foi colocado no subcutâneo, e o fechamento foi realizado por planos com pontos separados.

O procedimento demorou cerca de 2 horas. Logo após a cirurgia, o paciente foi extubado e reavaliado na sala de recuperação anestésica. Não apresentou déficits adicionais em relação ao exame pré-operatório.

0 paciente permaneceu internado por 8 dias e recebeu alta com o quadro neurológico semelhante ao da admissão. Não apresentou fistula liquórica nem infecção da ferida operatória. Não apresentou sinais clínicos de lesão da artéria vertebral. Após 3 meses de alta, deambulava com muletas, com força de grau $\mathrm{V}$ em ambos os membros superiores e com força de grau II no membro inferior esquerdo. A tomografia de controle mostrou ausência de corpos estranhos ou fragmentos ósseos no canal vertebral, com lordose cervical preservada (-Figs. 5 e $\mathbf{6}$ ). 0 exame de raios $\mathrm{X}$ dinâmico não demonstrou luxações. Foram

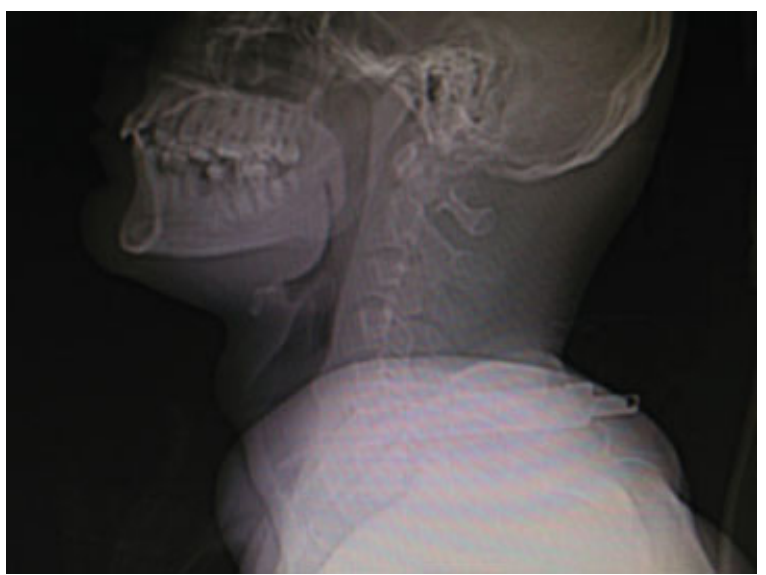

Fig. 2 Radiografia da admissão em perfil, evidenciando arma branca na coluna cervical posterior, no nível de C6-C7. 


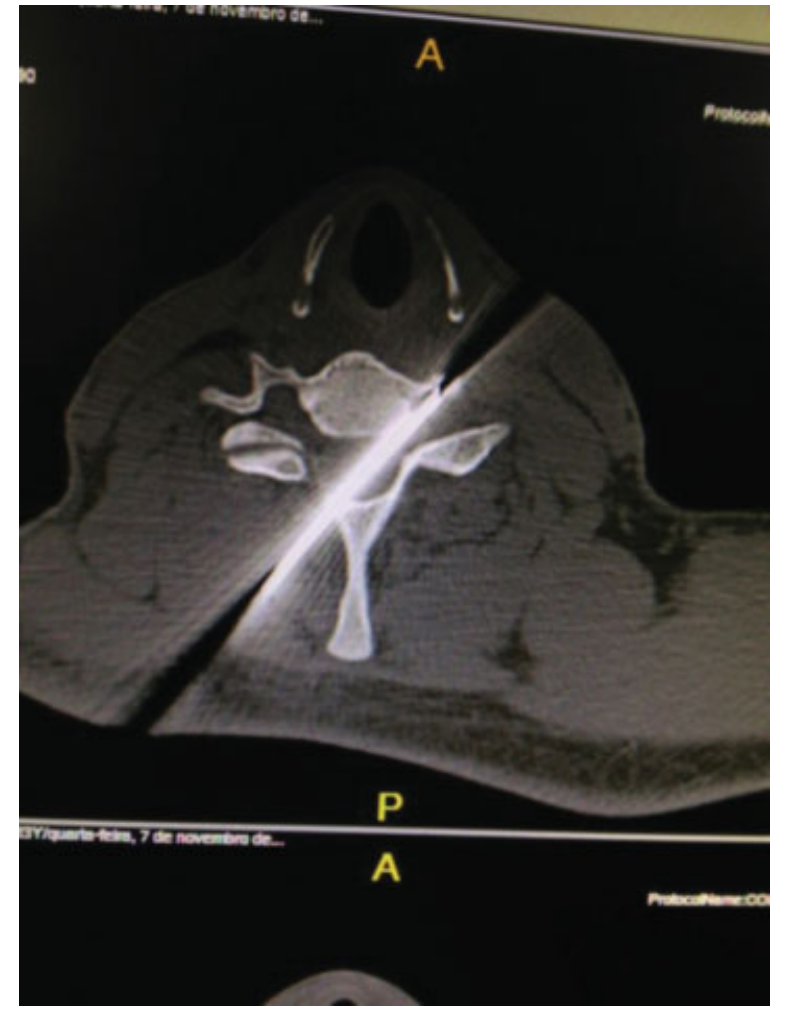

Fig. 3 Tomografia da coluna cervical pré-operatória em corte axial, mostrando trajeto da arma através da vértebra, com penetração do canal vertebral até o forame da artéria vertebral esquerda.

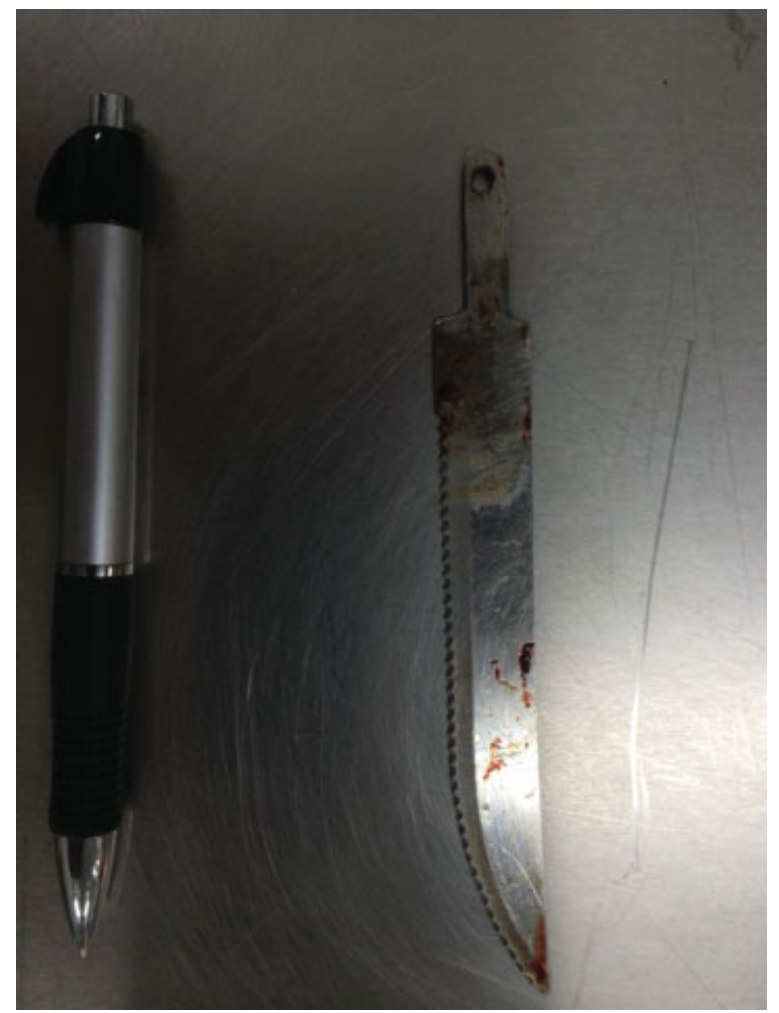

Fig. 4 Corpo estranho retirado após laminectomia.

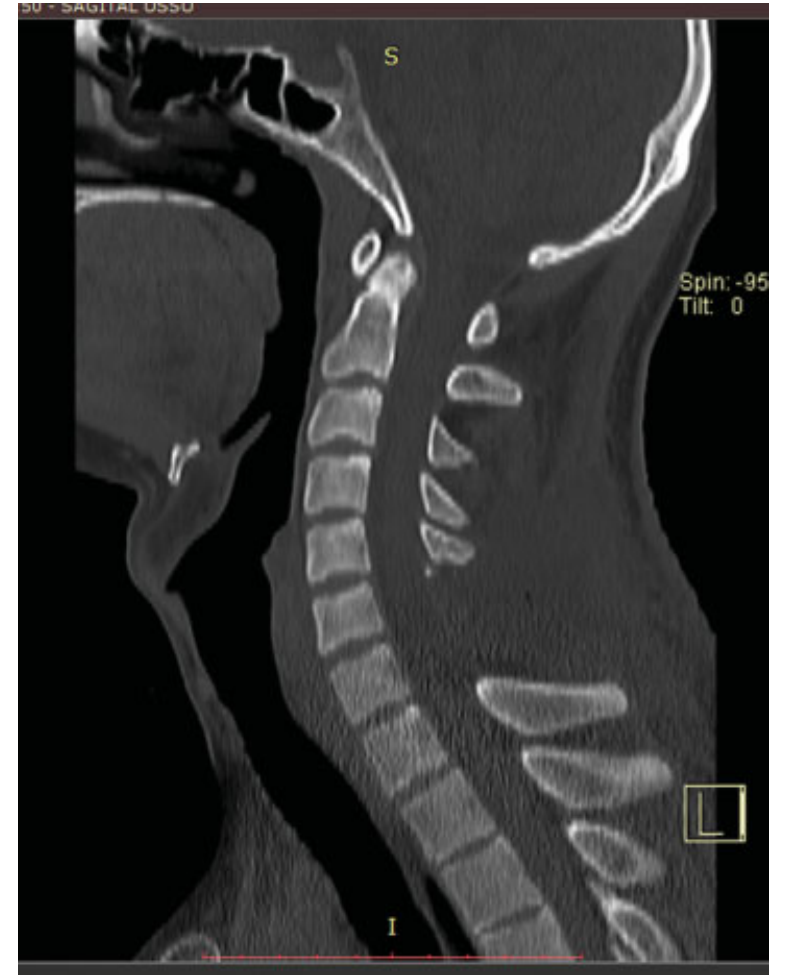

Fig. 5 Tomografia da coluna cervical pós-operatória.

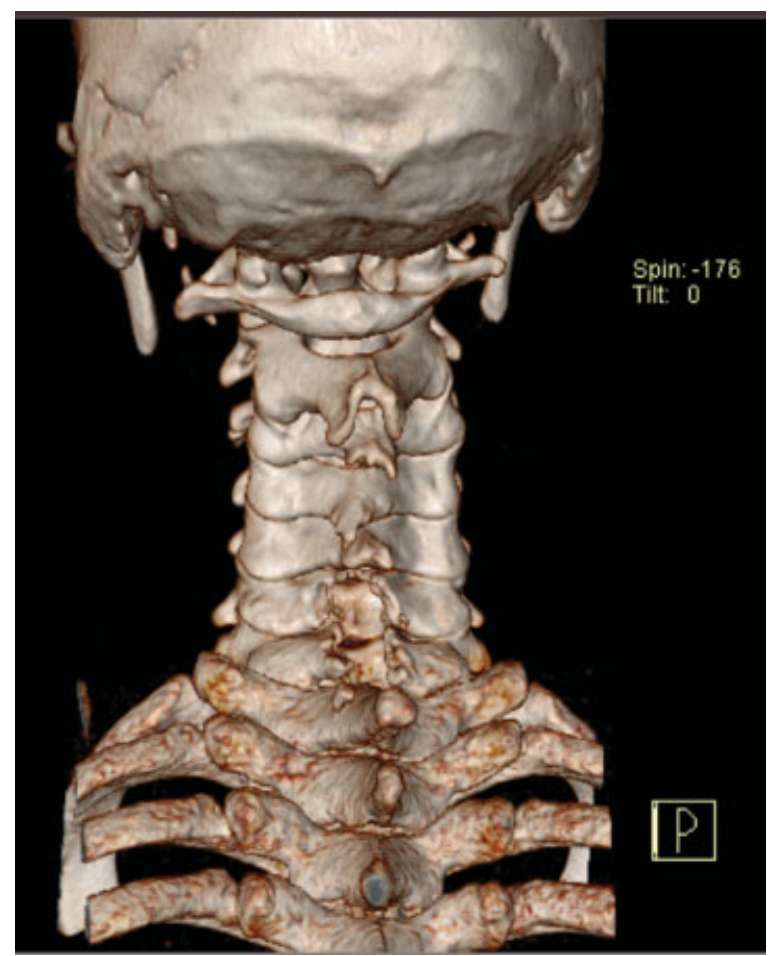

Fig. 6 Tomografia com reconstrução 3D pós-operatória. 
solicitadas arteriografia e ressonância magnética da coluna cervical 3 meses após a alta, mas o acompanhamento foi perdido, e o paciente não mais retornou ao ambulatório.

\section{Discussão}

O tratamento cirúrgico para o TRM penetrante tem como objetivos principais evitar progressão do déficit neurológico e corrigir eventuais instabilidades da coluna. $O$ cirurgião deve ser capaz de realizar o tratamento antes que o paciente desenvolva o déficit neurológico completo. ${ }^{6}$

As indicações de abordagem cirúrgica de um paciente com TRM por arma branca são: corpo estranho retido, presença de fístula liquórica, piora progressiva do quadro neurológico, 5,9,10 herniações da medula, ${ }^{14}$ hematoma epidural agudo, ${ }^{15}$ lesões vasculares ${ }^{16}$ ou evidências de compressões medulares. ${ }^{8,10}$

As artérias vertebrais estão localizadas dentro do forame vertebral, protegidas pelos processos transversos de C6 a C2 ${ }^{17,18}$ Sua lesão é pouco frequente. Ocorre em $7,4 \%$ das perfurações provocadas por arma branca na coluna cervical, contra $1 \%$ das decorrentes de armas de fogo. ${ }^{19-21}$ O melhor exame para sua detecção é o cateterismo angiográfico. ${ }^{22-24}$

Aproximadamente $50 \%$ dos pacientes com lesão motora incompleta decorrente de TRM por FAB apresentam síndrome de Brown-Séquard. ${ }^{4}$ Destes, apenas uma pequena parte cursa com todas as características da síndrome, composta por diminuição da sensibilidade termoálgica em um dimídio e paresia com diminuição da sensibilidade vibratória e postural contralaterais. ${ }^{25} \mathrm{O}$ paciente do nosso estudo apresentava todas as características desta síndrome.

Lesões medulares por arma branca comumente determinam déficits neurológicos imediatos a deterioração neurológica tardia e são observadas em uma minoria de pacientes (4,9\%). Suas possíveis causas são a siringomielia, aracnoidite, degeneração microcística, herniação da medula por defeito dural, formação de abscessos, granulomas ou incrustação de ferro na medula. ${ }^{15,23,24,26-30}$

Um estudo multicêntrico prospectivo, que acompanhou a evolução do déficit neurológico de 32 pacientes vítimas de TRM por $F A B$, verificou que, daqueles que apresentavam lesões incompletas, mais da metade retornou ao emprego. Esse estudo mostrou também que $14 \%$ dos pacientes evoluíram com melhora completa do déficit motor após 3 a 9 meses. ${ }^{4}$

Peacock et al, ${ }^{10}$ na mais longa série de casos sobre o assunto, constataram que dois terços dos pacientes tiveram recuperação funcional positiva. Resultados semelhantes foram verificados por outros autores. ${ }^{3,4}$

Após 3 meses de recuperação, o paciente do caso descrito foi capaz de deambular com muletas e recuperou completamente o déficit do membro superior. Apresentava força de grau III no membro inferior esquerdo e disestesias no membro inferior direito.

\section{Conclusão}

Pacientes com lesão medular incompleta por arma branca retida na coluna apresentam bom prognóstico se forem tratados precocemente. O objetivo da cirurgia é a retirada do objeto retido, sem provocar déficit motor ou sensitivo adicional, com correção do defeito dural e de eventuais instabilidades.

\section{Referências}

1 Del Bel EA, Silva CA, Mlasinic MO. Trauma raquimedular. Rev Coluna/Columna 2009;8(4):441-449

2 Brito LM, Chein MB, Marinho SC, Duarte TB. Epidemiological evaluation of victims of spinal cord injury. Rev Col Bras Cir 2011;38(5):304-309

3 Velmahos GC, Degiannis E, Hart K, Souter I, Saadia R. Changing profiles in spinal cord injuries and risk factors influencing recovery after penetrating injuries. J Trauma 1995;38(3): 334-337

4 Waters RL, Sie I, Adkins RH, Yakura JS. Motor recovery following spinal cord injury caused by stab wounds: a multicenter study. Paraplegia 1995;33(2):98-101

5 Rubin G, Tallman D, Sagan L, Melgar M. An unusual stab wound of the cervical spinal cord: a case report. Spine $2001 ; 26(4)$ : 444-447

6 Lopez AC. Tratado de Clínica Médica. Vol. II. 2ª ed. Roca; 2009

7 Baghai P, Sheptak PE. Penetrating spinal injury by a glass fragment: case report and review. Neurosurgery 1982;11(3):419-422

8 Thakur RC, Khosla VK, Kak VK. Non-missile penetrating injuries of the spine. Acta Neurochir (Wien) 1991;113(3-4):144-148

9 Lipschitz R, Block J. Stab wounds of the spinal cord. Lancet 1962; 2(7248):169-172

10 Peacock WJ, Shrosbree RD, Key AG. A review of 450 stabwounds of the spinal cord. S Afr Med J 1977;51(26):961-964

11 Lipschitz R. Stab wound of the spinal cord. Br J Med 1978; 1:1093-1094

12 Cabezudo JM, Carrillo R, Areitio E, García de Sola R, Vaquero J. Accidental stab wound of the cervical spinal cord from in front. Acta Neurochir (Wien) 1980;53(3-4):175-180

13 Defino HLA. Trauma raquimedular. Medicina Ribeirão Preto 1999; 32:388-400

14 Lee ST, Lui TN, Jeng CM. Spinal cord herniation after stabbing injury. Br J Neurosurg 1997;11(1):84-86

15 Shahlaie K, Chang DJ, Anderson JT. Nonmissile penetrating spinal injury. Case report and review of the literature. J Neurosurg Spine 2006;4(5):400-408

16 Jallo GI. Neurosurgical management of penetrating spinal injury. Surg Neurol 1997;47(4):328-330

17 Ahn JY, Kim OJ, Song WS, Lee BH, Joo JY. Guglielmi detachable coils embolization of a penetrating vertebral artery injury: a case report. J Trauma 2003;55(6):1171-1174

18 Mwipatayi BP, Jeffery P, Beningfield SJ, Motale P, Tunnicliffe J, Navsaria PH. Management of extra-cranial vertebral artery injuries. Eur J Vasc Endovasc Surg 2004;27(2):157-162

19 Vinces FY, Newell MA, Cherry RA. Isolated contralateral vertebral artery injury in a stab wound to the neck. J Vasc Surg 2004;39(2): 462-464

20 Hindman BJ, Palecek JP, Posner KL, et al. Cervical spinal cord, root, and bony spine injuries: a closed claims analysis. Anesthesiology 2011;114(4):782-795

21 Seo BR, Lee JK, Lee JH, Kim SH. An unusual stab wound causing a traumatic pseudomeningocele at the craniocervical junction. J Clin Neurosci 2009;16(10):1365-1367

22 Williams DT, Chang DL, DeClerck MP. Penetrating spinal cord injuries with retained canal fragments. CJEM 2009;11(2): 172-173

23 Manzone P, Domenech V, Forlino D. Stab injury of the spinal cord surgically treated. J Spinal Disord 2001;14(3):264-267

24 Lloyd RV, O'Flynn PE, Jones NS. Access to the traumatized vertebral artery: an unusual approach. J Laryngol Otol 1999; 113(1):76-78 
25 Koehler PJ, Endtz LJ. The Brown-Séquard syndrome. True or false? Arch Neurol 1986;43(9):921-924

26 Wolf SM. Delayed traumatic myelopathy following transfixion of the spinal cord by a knife blade. Case report. J Neurosurg 1973; 38(2):221-225

27 Rout D, Kak VK, Gulati DR. Stab injuries of the spinal cord. Neurol India 1978;26(4):186-189
28 Marshall LF, Knowlton S, Garfin SR, et al. Deterioration following spinal cord injury. A multicenter study. J Neurosurg 1987;66(3):400-404

29 Berrington NR. Posttraumatic spinal cord tethering. Case report. J Neurosurg 1993;78(1):120-121

30 Silberstein M, Hennessy O. Cystic cord lesions and neurological deterioration in spinal cord injury: operative considerations based on magnetic resonance imaging. Paraplegia 1992;30(9):661-668 